\title{
Progesterone influences cytoplasmic maturation in porcine oocytes developing in vitro
}

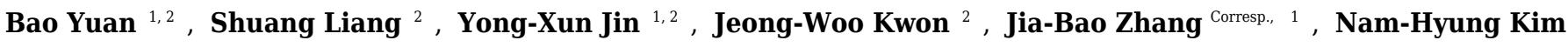 \\ Corresp. 1,2 \\ 1 Department of Laboratory Animal, College of Animal Sciences, Jilin university, Changchun, Jilin, P.R.China \\ 2 Molecular Embryology Laboratory, Department of Animal Sciences, Chungbuk National University, Cheongju, Chungbuk, Korea \\ Corresponding Authors: Jia-Bao Zhang, Nam-Hyung Kim \\ Email address: zjb515@126.com, nhkim@chungbuk.ac.kr
}

Progesterone (P4), an ovarian steroid hormone, is an important regulator of female reproduction. In this study, we explored the influence of progesterone on porcine oocyte nuclear maturation and cytoplasmic maturation and development in vitro. We found that the presence of P4 during oocyte maturation did not inhibit polar body extrusions but significantly increased glutathione and decreased reactive oxygen species (ROS) levels relative to that in control groups. The incidence of parthenogenetically activated oocytes that could develop to the blastocyst stage was higher $(p<0.05)$ when oocytes were exposed to P4 as compared to that in the controls. Cell numbers were increased in the P4treated groups. Further, the P4-specific inhibitor mifepristone (RU486) prevented porcine oocyte maturation, as represented by the reduced incidence $(p<0.05)$ of oocyte first polar body extrusions. RU486 affected maturation promoting factor(MPF) activity and maternal mRNA polyadenylation status. In general, these data show that P4 influences the cytoplasmic maturation of porcine oocytes, at least partially, by decreasing their polyadenylation, thereby altering maternal gene expression. 
1 Progesterone influences cytoplasmic maturation in porcine oocytes developing in vitro

2

3 Bao Yuan ${ }^{1,2 \#, ~ S h u a n g ~ L i a n g ~}{ }^{2 \#}$, Yong-Xun Jin ${ }^{1}$, Jeong-Woo Kwon², Jia-Bao Zhang ${ }^{*}$, Nam4 Hyung Kim ${ }^{1,2 *}$

5

6

7

${ }^{1}$ Department of Laboratory Animal, College of Animal Sciences, Jilin university, Changchun, Jilin, P.R.China

${ }^{2}$ Molecular Embryology Laboratory, Department of Animal Sciences, Chungbuk National University, Cheongju, Chungbuk, Korea

\#These authors contributed equally to this work.

*Correspondence:

Nam-Hyung Kim ${ }^{1,2}$

Molecular Embryology Laboratory, Department of Animal Sciences, Chungbuk National

University, Cheongju 361-763, Korea

E-mail: nhkim@,chungbuk.ac.kr

Jia-Bao Zhang ${ }^{1}$

Department of Laboratory Animal, College of Animal Sciences, Jilin University, Changchun, Jilin 130062, P.R. China

E-mail: zjb515@126.com 
25

26

27

\section{ABSTRACT}

Progesterone (P4), an ovarian steroid hormone, is an important regulator of female reproduction. In this study, we explored the influence of progesterone on porcine oocyte nuclear maturation and cytoplasmic maturation and development in vitro. We found that the presence of P4 during oocyte maturation did not inhibit polar body extrusions but significantly increased glutathione and decreased reactive oxygen species (ROS) levels relative to that in control groups. The incidence of parthenogenetically activated oocytes that could develop to the blastocyst stage was higher $(p$ $<0.05)$ when oocytes were exposed to P4 as compared to that in the controls. Cell numbers were increased in the P4-treated groups. Further, the P4-specific inhibitor mifepristone (RU486) prevented porcine oocyte maturation, as represented by the reduced incidence $(p<0.05)$ of oocyte first polar body extrusions. RU486 affected maturation promoting factor (MPF) activity and maternal mRNA polyadenylation status. In general, these data show that P4 influences the cytoplasmic maturation of porcine oocytes, at least partially, by decreasing their polyadenylation, thereby altering maternal gene expression. 


\section{INTRODUCTION}

In mammals, follicle-stimulating hormone (FSH) and luteinizing hormone (LH), which are secreted by the pituitary gland, are primarily responsible for follicular growth and ovulation. Thus, FSH and/or LH are usually added to in vitro maturation (IVM) medium. In the pig, previous studies have shown that the addition of FSH to IVM medium can promote cumulus expansion and increase the ratio of oocytes undergoing germinal vesicle breakdown (GVBD) and/or achieving metaphase II (MII) (Algriany et al. 2004; Su et al. 1999). Furthermore, FSH stimulates cumulus cell expansion and increases progesterone concentration in the follicle in pigs (Blaha et al. 2015).

During follicular development, the concentrations of steroid hormones change, and ratios of progesterone and estradiol may affect oocyte maturation. In rhesus monkeys and humans, high ratios of P4 to estrogen seem to be related to high rates of embryogenesis and frequency of pregnancy (Dumesic et al. 2003; Wagner et al. 2012). High ratios of progesterone and estradiol in the IVM medium of oocytes promote developmental capacity in monkeys (Zheng 2007; Zheng et al. 2003). Similarly, in cattle, progesterone in the maturation medium improves the frequency of development to the blastocyst stage (Ryan et al. 1992). In porcine oocytes, adding P4 to the IVM medium accelerates meiosis resumption (Eroglu 1993; Sirotkin \& Nitray 1992) and enhances IVM via follicular fluid and embryonic development. Mifepristone (RU486) is an 11ß-dimethyl-aminophenyl derivative of norethindrone with a high affinity for progesterone (Belanger et al. 1981). RU486 effectively prevents $\mathrm{P} 4$ receptors activity in porcine placentae, and can terminate pregnancy (Hapangama \& Neilson 2009). Previously, RU486 has been shown to suppress the cumulus expansion and meiotic maturation of porcine cumulus-oocyte complexes (COCs) in culture (Shimada et al. 2004; Yamashita et al. 2010).

Although studies indicate that P4 improves oocyte nuclear maturation, the underlying mechanism of progesterone's positive effect on the oocyte cytoplasm during in vitro maturation has not yet been determined. There are multiple ways to evaluate the quality of MII stage oocytes and early embryos. Intracellular levels of ROS and GSH are critical factors that influence oocyte maturation and subsequent embryo development (Evans et al. 2004; Kang et al. 2016). Maturation 
68

promoting factor (MPF) is the principal regulatory molecule driving meiotic progression during oocyte maturation (Lin et al. 2014). The poly(A) tail (PAT) length of the MPF gene influences further embryonic development (Zhang et al. 2010). Apoptosis-related genes and cell apoptotic rates reflect the quality of the blastocysts (Han et al. 2016).

In this study, progesterone was added during the in vitro maturation of pig oocytes. The beneficial effect of progesterone on oocyte quality was investigated by evaluating early embryonic development in porcine oocytes after progesterone supplementation during in vitro maturation. Furthermore, various functional features, such as ROS levels, GSH levels, maternal gene expression, polyadenylation levels, apoptosis levels in blastocysts, and p34cdc2 kinase activity in oocytes were evaluated and compared after progesterone supplementation.

\section{MATERIALS AND METHODS}

This study was carried out in strict accordance with the Guide for the Care and Use of Laboratory Animals of Jilin University. Animal procedures were conducted following the protocol (20151207) approved by the Animal Care \& Welfare Committee of Jilin University. All chemicals used were purchased from the Sigma-Aldrich Chemical Company (St. Louis, MO, USA) unless otherwise stated.

\section{Collection and IVM of porcine oocytes}

Porcine COCs were recovered from follicles 3-6 $\mathrm{mm}$ in diameter in porcine ovaries and washed three times with TL-HEPES [with $0.05 \mathrm{~g} / \mathrm{L}$ gentamycin and $1 \mathrm{~g} / \mathrm{L}$ polyvinyl alcohol (PVA) added]. The collected COCs were matured in IVM medium for $44 \mathrm{~h}$ at $38.5^{\circ} \mathrm{C}$ in $5 \% \mathrm{CO}_{2}$ and humidified air. Normal IVM medium is comprised of tissue culture medium 199 (Gibco) supplemented with $0.1 \mathrm{~g} / \mathrm{L}$ sodium pyruvate, $0.6 \mathrm{mM}$ L-cysteine, $10 \mathrm{ng} / \mathrm{mL}$ epidermal growth factor, $10 \%$ porcine follicular fluid (PFF) (v/v), $10 \mathrm{IU} / \mathrm{mL} \mathrm{LH}$, and $10 \mathrm{IU} / \mathrm{mL}$ FSH. We also tried using normal IVM medium that was not supplemented with PFF, LH, or FSH.

Based on a previous study (Salehnia \& Zavareh 2013; Shimada \& Terada 2002), different 
95

96

concentrations of progesterone $(0 \mu \mathrm{M}, 10 \mu \mathrm{M}$, or $100 \mu \mathrm{M})$ and RU486 $(0 \mu \mathrm{M}, 10 \mu \mathrm{M}$, or $25 \mu \mathrm{M})$ were added to the culture media. After IVM, the COCs were washed in TL-HEPES (with hyaluronidase $[1 \mathrm{mg} / \mathrm{mL}]$ and PVA $[0.1 \%, \mathrm{v} / \mathrm{v}]$ added) to remove cumulus cells. The oocytes were added to normal TL-HEPES and the oocytes in which the first polar bodies had discharged were selected for further studies.

\section{Measurement of MII oocyte ROS and GSH levels}

To detect the ROS and GSH levels, MII stage oocytes were sampled in medium with added P4 $(100 \mu \mathrm{M})$ or RU-486 $(25 \mu \mathrm{M})$ for determination of their intracellular ROS and GSH levels. For detection of the ROS levels, the oocytes were incubated with $10 \mu \mathrm{M} \mathrm{H}_{2}$ DCFDA for 15 min (green fluorescence, UV filters, $460 \mathrm{~nm}$ ). For detection of the GSH levels, the oocytes were incubated with $10 \mu \mathrm{M}$ CMF2HC (Invitrogen) for $15 \mathrm{~min}$ (blue fluorescence, UV filters, $370 \mathrm{~nm}$ ). The same procedures were followed for all groups of oocytes, including incubation, rinse, mounting and imaging. Image $\mathrm{J}$ software was used to analyze the fluorescence intensities of the oocytes. Three independent experiments were performed.

\section{Analysis of poly(A) tail lengths by polymerase chain reaction (PCR)}

To detect the maternal transcripts poly(A) tail length, PAT assay was performed as described previously (Zhang et al. 2010). Briefly, total mRNAs from MII pig oocytes were reverse transcribed with anchored oligo(dT) primer (Table 1) (Salles \& Strickland 1999). PCR was performed using anchored oligo(dT) primer and PAT-Cdc2 primer to test the maternal transcripts (Table 1). The adjustment PCR program was run for 5 min at $95{ }^{\circ} \mathrm{C}$, followed by 35 cycles of 20 s at $94{ }^{\circ} \mathrm{C}, 45 \mathrm{~s}$ at $60{ }^{\circ} \mathrm{C}, 45 \mathrm{~s}$ at $72{ }^{\circ} \mathrm{C}$, and finally, for an extension of $3 \mathrm{~min}$ at $72{ }^{\circ} \mathrm{C}$. A $3.0 \%$ agarose gel electrophoresis was performed to analyze the PCR products.

\section{MII oocyte MPF activity assay}


121

122

123

124

125

126

127

128

129

130

131

132

133

134

135

136

137

138

139

140

141

142

143

144

145

146

The Cdc2/Cdk1 Kinase Assay Kit (MBL, Nagoya, Japan) was used to quantify p34cdc2 kinase activity (Lin et al. 2014; Zhang et al. 2010). Briefly, 30 oocytes were washed three times in sample buffer. Oocyte extract $(5 \mu \mathrm{L})$ was mixed with kinase assay buffer $(45 \mu \mathrm{L})$. The mixture was placed in an incubator at $30{ }^{\circ} \mathrm{C}$ for $30 \mathrm{~min}$. The reaction was terminated by $200 \mu \mathrm{L}$ ethylene glycol tetraacetic acid $(50 \mathrm{mM})$. The OD value was read at $492 \mathrm{~nm}$. Three independent experiments were performed.

\section{Parthenogenetic activation and in vitro culture of pig oocytes}

After $42 \mathrm{~h}$ of maturation, MII stage oocytes were selected. Denuded oocytes with homogeneous cytoplasm were selected and then gradually equilibrated in activation solution by a $1.0 \mathrm{kV} / \mathrm{cm}$ electric pulse for $60 \mu \mathrm{s}$. Activated oocytes were hatched in PZM-5 medium with $2 \mathrm{mM}$ cytochalasin B for 3 h. Next, approximately 40-50 post-activation oocytes were cultured in PZM-5 for 7 days, and embryos were cultured at $38.5{ }^{\circ} \mathrm{C}$ in $5 \% \mathrm{CO}_{2}$ (Kwon et al. 2015).

\section{Real-time reverse transcription PCR}

Total mRNA extraction and cDNA synthesis were performed as previously described (Lee et al. 2014). Briefly, 50 MII oocytes or 20 blastocysts were used to extract mRNA with a Dynabeads mRNA Direct Kit, following reverse transcription of the mRNA by oligo(dT) 12-18 primer with a SuperScript Reverse Transcriptase Kit. The primers used for the real-time PCR (RT-PCR) are listed in Table 1. The reaction was performed in a Bio-Rad CFX PCR machine. The $2^{-\Delta \Delta C t}$ method was followed to analyze gene expression (Livak \& Schmittgen 2001). The control gene was

glyceraldehyde 3-phosphate dehydrogenase $(G A P D H)$. Three independent experiments were performed in triplicate.

\section{Confocal microscopy and counting the number of nuclei per blastocyst}

Counting methods were described previously (Liang et al. 2015). Briefly, the blastocysts were 
147 fixed in 3.7\% paraformaldehyde prepared in PBS-PVA for $30 \mathrm{~min}$, then washed in PBS washing 148 solution and $0.3 \%$ Triton X-100 for $1 \mathrm{~h}$. After being washed twice in PBS, the blastocysts were 149 put in fluorescein-conjugated dUTP and terminal deoxynucleotidyl transferase enzyme in the dark 150 for $1 \mathrm{~h}$. Next, they were incubated with $10 \mu \mathrm{g} / \mathrm{mL}$ Hoechst 33342 and $50 \mathrm{mg} / \mathrm{mL}$ RNase A for 1

151 152

153

154 155

\section{Statistical analyses}

All the experiment results were analyzed by one-way ANOVA and a Chi-square test to determine the $p$-value, using IBM SPSS Statistics 19 software. A $p$-value $<0.05$ was considered statistically significant.

\section{RESULTS}

\section{Effects of P4 and RU486 IVM on porcine oocytes}

To evaluate the effects of P4 and RU486 on porcine oocyte maturation, porcine oocytes were treated with various concentrations of P4 $(0,10$, or $100 \mu \mathrm{M})$ and RU486 $(0,10$, or $25 \mu \mathrm{M})$, and their polar body extrusions were examined. The oocytes were randomly divided into three groups: normal IVM medium with added P4; IVM medium (without FSH, LH, and PFF) with added P4; and normal IVM medium with added RU486 (Fig. 1). In both the normal IVM medium and IVM medium (without FSH, LH, and PFF) groups, adding different concentrations of P4 had no effect on MII oocyte rate $(p>0.05)$. However, in the normal IVM medium with added RU486 group, adding $10 \mu \mathrm{M}$ or $25 \mu \mathrm{M}$ RU486 significantly reduced the MII oocyte rate $(p<0.01)$.

\section{Effects of P4 and RU486 on intracellular levels of ROS and GSH}

To determine the mechanism by which P4 and RU486 influence porcine oocyte maturation, the levels of ROS and GSH were examined. Significant decreases in the levels of ROS and GSH were 
173

174

175

176

177

178

179

180

181

182

183

184

185

186

187

188

189

190

191

192

193

194

195

196

197

198

199

observed in porcine oocytes after IVM. The levels of ROS were significantly lower in the $100-\mu \mathrm{M}$ P4-treated oocytes (4.95 \pm 1.57 pixels/oocyte; Fig. $2 \mathrm{~B})$, but higher in the $25-\mu \mathrm{M}$ RU486-treated oocytes $(15.48 \pm 3.67$ pixels/oocyte; Fig. 2 C) than in the control group ( $8.65 \pm 1.99$ pixels/oocyte; Fig. 2A). The levels of GSH were higher significantly in the $100-\mu \mathrm{M}$ P4-treated oocytes $(69.29 \pm$ 4.81 pixels/oocyte; Fig. 2E), but lower in the $25-\mu \mathrm{M}$ RU486-treated oocytes $(47.40 \pm 8.28$ pixels/oocyte; Fig. 2F) than in the control group (63.78 \pm 7.05 pixels/oocyte; Fig. 2D).

\section{Effects of P4 and RU486 on maternal gene expression, polyadenylation levels, and p34cdc2 kinase activity}

Maternal gene expression is an important biological process in oocyte maturation and early embryo development. We examined the expression of maternal genes $c d c 2$ and $c y c l i n b 1$ (regulatory subunits of MPF). After treatment of the oocytes with P4, their $c d c 2$ and $c y c l i n b 1$ mRNA levels increased, but decreased after $44 \mathrm{~h}$ of treatment with RU486 (Fig. 3A). We also analyzed the $c d c 2$ gene poly(A) tail length at the MII stage (Fig. 3B). In this treatment, $25 \mu \mathrm{M}$ RU486 affected the maternal mRNA polyadenylation status by shortening signal smears. In 100$\mu \mathrm{M}$ P4-treated oocytes, $c d c 2$ underwent polyadenylation at MII; there were no significant differences from that in the control group. When COCs were IVM-treated with RU486, the activation of $p 34 c d c 2 a$ decreased at the MII stage as compared with that in the control and P4treated groups (Fig. 3C).

\section{Effects of P4 and RU486 during IVM on embryo development}

To determine whether P4 or RU486 treatment during IVM influences subsequent embryonic development, porcine oocytes were activated and their in vitro development examined (Fig. 4). In normal medium, the addition of different concentrations of $\mathrm{P} 4$ had no effect on the oocyte blastocyst rate $(p>0.05)$. In medium without $\mathrm{FSH}, \mathrm{LH}$, and PFF, the blastocyst rate was significantly higher in the $100-\mu \mathrm{M}$ P4-treated group than in the other groups $(50.56 \%$ vs. $42.30 \%$, $38.36 \% ; p<0.05)$. However, adding $10 \mu \mathrm{M}$ or $100 \mu \mathrm{M}$ RU486 significantly reduced the blastocyst 
200

201

202

203

204

205

rate $(34.44 \%, 32.26 \%$ vs. $47.16 \%)$.

\section{TUNEL assay at the blastocyst stage}

DNA fragments generated by apoptotic nicking of genomic DNA were measured in individual embryos by TUNEL assay. The apoptotic rate at the blastocyst stage was significantly higher in the RU486-treated group than in the P4-treated group (Fig. 5A and 5C). The number of blastocysts was higher in the P4-treated group than in the control group (42.1 \pm 3.9 vs. $47.5 \pm 5.3)$; however, no significant difference was found between the RU486-treated and control groups (Fig. 5B). To further explore the manner in which P4 and RU486 influence the incidence of apoptotic cells in parthenogenetic blastocysts, the expression of the apoptosis-related genes Bcl2, Bax, and Casp3 was evaluated in parthenogenetic blastocysts. Compared with that in the non-treated group, the expression of Casp3 and Bax mRNA was significantly higher and the expression of $B c l-2$ mRNA was significantly lower in the RU486-treated group (Fig. 6). 
215

216

217

218

219

220

221

222

223

224

225

226

227

228

229

230

231

232

233

234

235

236

237

238

239

240

241

\section{DISCUSSION}

Different maturation media have been reported to have distinct effects on oocyte IVM (Wang et al. 1997), and oocytes are more susceptible to compromised developmental potency under suboptimal conditions (Zhou et al. 2012). Previous studies have shown that the level of progesterone produced by cumulus cells is increased by the stimulation of LH and FSH and influences porcine oocyte maturation (Shimada \& Terada 2002). However, the mechanism by which P4 influences porcine oocyte maturation is not clear. In this study, we examined the effects of P4 and RU486 on IVM of porcine COCs. Adding P4 to normal culture medium did not improve COC maturation and developmental ability. Adding P4 to the culture medium without PFF, LH, and FSH did not improve the maturation of porcine COCs, but did increase their developmental ability when $100 \mu \mathrm{M}$ P4 was added. Adding $10 \mu \mathrm{M}$ or $25 \mu \mathrm{M}$ RU486 significantly reduced the maturation rate of porcine oocytes and their developmental ability.

Studies indicate that progesterone is produced by the cumulus cells during IVM (Shimada \& Terada 2002), and co-culture with cumulus cells significantly increases maturation rate and blastocyst formation of denuded oocytes during IVM in sheep (Kyasari et al. 2012), mouse (Jiao et al. 2013), porcine (Yoon et al. 2015), goat (Wang et al. 2011), human (Combelles et al. 2005), and rat (Jiao et al. 2016). However, it is not clear whether progesterone produced by the cumulus cell monolayer is also positively involved in these co-culture systems. Our results agreed with those of previous reports on the function of P4 in bovine oocyte maturation (Aparicio et al. 2011). The results appear to show a positive role for P4 in MII-stage oocyte quality. P4 also improves in vitro cytoplasmic maturation in monkey and canine oocytes (Vannucchi et al. 2006; Zheng et al. 2003). In contrast to the results of the present study, adding P4 to the medium did not enhance the maturation of mouse germinal vesicle $(\mathrm{GV})$ oocytes or their developmental ability (Zavareh et al. 2009). P4 supplementation of IVM culture systems did not affect the rate of IVM of bovine oocytes, and addition of $\mathrm{P} 4$ to the IVF medium did not improve the rate of cleavage stage embryos (Carter et al. 2010). Our results were similar to those found in porcine, bovine, and primate oocytes: P4 didn't increase the percentages of oocyte maturation (Karlach 1986; Nagyova et al. 
242 2014; Ryan et al. 1999; Zheng et al. 2003). However, the progesterone antagonist (RU486) could

243

244

245

246

247

248

249

250

251

252

253

254

255

256

257

258

259

260

261

262

263

264

265

266

267

268 not reverse the repression effect of progesterone on mouse oocyte maturation (Zavareh et al. 2009).

ROS are known to be mediators of caspase-dependent cell death (Jang et al. 2013; Le Bras et al. 2005). Previous studies have demonstrated that progesterone possesses antioxidant properties, forming scavenging rings of ROS in cancer cells and increasing superoxide dismutase (SOD) activity in human endometrial stromal cells (Matsuoka et al. 2010; Nguyen \& Syed 2011). Progesterone $(0.5 \mu \mathrm{M})$ protects mouse pancreatic islets against $\mathrm{H}_{2} \mathrm{O}_{2}$-induced oxidative stress and leads to decreased ROS production (Ahangarpour et al. 2014). In addition, other studies show that P4 has the ability to increase ROS levels in MCF-7 cells (Azeez et al. 2015). However, ROS signaling does not show any apoptotic effect of RU486 treatment in U937 cells (Jang et al. 2013). It has been shown that mitochondrial dysfunction is directly responsible for impaired developmental potential of oocytes (Dai et al. 2015). RU486, a P4 receptor antagonist, almost completely blocked the effect of progesterone on ROS protection, indicating that ROS overproduction is mediated via GC receptors.

Adding an antioxidant to the medium influences maturation rate and developmental ability. Previous studies on cattle, goats, and pigs have shown that antioxidants improve embryo developmental ability and increase embryonic GSH levels (Droge 2002; Mukherjee et al. 2014). In the present study, GSH activity in MII oocytes was decreased significantly when RU486 was added during IVM.

MPF signals are critical for oocyte maturation. Moreover, $c d c 2$ and $c y c l i n B 1$ are important genes of maturation promoting factor (Zhao et al. 2014). In previous studies, we found that in good-quality MII-stage oocytes, cyclinb1 and $c d c 2$ gene expression was increased (Lin et al. 2014; Zhang et al. 2010). In this study, we used P4-treated oocytes that had high expression of the $c d c 2$ gene. Examination of poly(A) clearly showed degradation of Cdc2 isoforms in MII oocytes (Zhang et al. 2010). In the present study, progesterone had no significant effect on the length of poly(A), and after RU486 treatment, poly(A) length decreased. Previous studies reported changes in MPF expression in porcine oocytes as they reactivated to enter MII (Lin et al. 2014). In the present 
269

270

271

272

273

274

275

276

277

278

279

280

281

study, the decline in MPF activity occurred following RU486 treatment.

In previous studies, high-quality oocytes showed lower expression levels of Bax mRNA, but higher levels of Bcl-2 mRNA (Yang \& Rajamahendran 2002). In the present study, we found that RU486 decreased oocyte quality and increased apoptosis in blastocysts; this is similar to the results of other studies where RU486 was used to promote susceptibility to apoptosis (Quirk et al. 2004). We hypothesize that the effect of P4 is to inhibit blastocyst apoptosis, but treatment with P4 only increases the number of blastocysts.

In conclusion, we focused on the effects of $\mathrm{P} 4$ on porcine oocyte maturation. Our results support the hypothesis that addition of P4 improves the viability of porcine oocytes and their in vitro developmental ability, at least partially, by decreasing their polyadenylation, thereby altering the expression of other maternal genes. 
282

283

284

285

286

287

288

289

290

291

292

293

294

295

296

297

298

299

300

301

302

303

304

305

306

307

308

309

310

311

312

313

314

315

316

317

318

319

320

321

322

\section{REFERENCES}

Ahangarpour A, Heidari H, Mard SA, Hashemitabar M, and Khodadadi A. 2014. Progesterone and cilostazol protect mice pancreatic islets from oxidative stress induced by hydrogen peroxide. Iran J Pharm Res 13:937-944.

Algriany O, Bevers M, Schoevers E, Colenbrander B, and Dieleman S. 2004. Follicle size-dependent effects of sow follicular fluid on in vitro cumulus expansion, nuclear maturation and blastocyst formation of sow cumulus oocytes complexes. Theriogenology 62:1483-1497.

Aparicio IM, Garcia-Herreros M, O'Shea LC, Hensey C, Lonergan P, and Fair T. 2011. Expression, regulation, and function of progesterone receptors in bovine cumulus oocyte complexes during in vitro maturation. Biol Reprod 84:910-921.

Azeez JM, Sithul H, Hariharan I, Sreekumar S, Prabhakar J, Sreeja S, and Pillai MR. 2015. Progesterone regulates the proliferation of breast cancer cells - in vitro evidence. Drug Des Devel Ther 9:5987-5999.

Belanger A, Philibert D, and Teutsch G. 1981. Regio and stereospecific synthesis of 11 beta-substituted 19norsteroids. Influence of 11 beta-substitution on progesterone receptor affinity - (1). Steroids 37:361-382.

Blaha M, Nemcova L, Kepkova KV, Vodicka P, and Prochazka R. 2015. Gene expression analysis of pig cumulusoocyte complexes stimulated in vitro with follicle stimulating hormone or epidermal growth factor-like peptides. Reprod Biol Endocrinol 13:113.

Carter F, Rings F, Mamo S, Holker M, Kuzmany A, Besenfelder U, Havlicek V, Mehta J, Tesfaye D, and Schellander K. 2010. Effect of elevated circulating progesterone concentration on bovine blastocyst development and global transcriptome following endoscopic transfer of in vitro produced embryos to the bovine oviduct. Biol Reprod 83:707-719.

Combelles CM, Fissore RA, Albertini DF, and Racowsky C. 2005. In vitro maturation of human oocytes and cumulus cells using a co-culture three-dimensional collagen gel system. Hum Reprod 20:1349-1358.

Dai J, Wu C, Muneri CW, Niu Y, Zhang S, Rui R, and Zhang D. 2015. Changes in mitochondrial function in porcine vitrified MII-stage oocytes and their impacts on apoptosis and developmental ability. Cryobiology 71:291-298.

Droge W. 2002. Free radicals in the physiological control of cell function. Physiol Rev 82:47-95.

Dumesic DA, Schramm RD, Bird IM, Peterson E, Paprocki AM, Zhou R, and Abbott DH. 2003. Reduced intrafollicular androstenedione and estradiol levels in early-treated prenatally androgenized female rhesus monkeys receiving follicle-stimulating hormone therapy for in vitro fertilization. Biol Reprod 69:12131219.

Eroglu A. 1993. Experimental studies on in vitro maturation of porcine oocytes. II. Effects of estradiol-17 beta and progesterone. Berl Munch Tierarztl Wochenschr 106:157-159.

Evans MD, Dizdaroglu M, and Cooke MS. 2004. Oxidative DNA damage and disease: induction, repair and significance. Mutation Research/Reviews in Mutation Research 567:1-61.

Han J, Wang QC, Zhu CC, Liu J, Zhang Y, Cui XS, Kim NH, and Sun SC. 2016. Deoxynivalenol exposure induces autophagy/apoptosis and epigenetic modification changes during porcine oocyte maturation. Toxicol Appl Pharmacol 300:70-76.

Hapangama D, and Neilson JP. 2009. Mifepristone for induction of labour. Cochrane Database Syst Rev:CD002865.

Jang JH, Woo SM, Um HJ, Park EJ, Min KJ, Lee TJ, Kim SH, Choi YH, and Kwon TK. 2013. RU486, a glucocorticoid receptor antagonist, induces apoptosis in U937 human lymphoma cells through reduction in 
mitochondrial membrane potential and activation of p38 MAPK. Oncol Rep 30:506-512.

Jiao GZ, Cao XY, Cui W, Lian HY, Miao YL, Wu XF, Han D, and Tan JH. 2013. Developmental potential of prepubertal mouse oocytes is compromised due mainly to their impaired synthesis of glutathione. PLoS One 8:e58018.

Jiao GZ, Cui W, Yang R, Lin J, Gong S, Lian HY, Sun MJ, and Tan JH. 2016. Optimized Protocols for In Vitro Maturation of Rat Oocytes Dramatically Improve Their Developmental Competence to a Level Similar to That of Ovulated Oocytes. Cell Reprogram 18:17-29.

Kang JT, Moon JH, Choi JY, Park SJ, Kim SJ, Saadeldin IM, and Lee BC. 2016. Effect of Antioxidant Flavonoids (Quercetin and Taxifolin) on In vitro Maturation of Porcine Oocytes. Asian-Australas J Anim Sci 29:352358.

Karlach V. 1986. The effect of FSH, LH, oestradiol-17 beta, and progesterone on cytoplasmic maturation of bovine follicular oocytes in vitro. Folia biologica 33:258-265.

Kwon J, Namgoong S, and Kim NH. 2015. CRISPR/Cas9 as tool for functional study of genes involved in preimplantation embryo development. PLoS One 10:e120501.

Kyasari OR, Valojerdi MR, Farrokhi A, and Ebrahimi B. 2012. Expression of maturation genes and their receptors during in vitro maturation of sheep COCs in the presence and absence of somatic cells of cumulus origin. Theriogenology 77:12-20.

Le Bras M, Clement MV, Pervaiz S, and Brenner C. 2005. Reactive oxygen species and the mitochondrial signaling pathway of cell death. Histol Histopathol 20:205-219.

Lee SK, Zhao MH, Kwon JW, Li YH, Lin ZL, Jin YX, Kim NH, and Cui XS. 2014. The association of mitochondrial potential and copy number with pig oocyte maturation and developmental potential. $J$ Reprod Dev 60:128-135.

Liang S, Zhao MH, Choi JW, Kim NH, and Cui XS. 2015. Scriptaid Treatment Decreases DNA Methyltransferase 1 Expression by Induction of MicroRNA-152 Expression in Porcine Somatic Cell Nuclear Transfer Embryos. PLoS One 10:e134567.

Lin ZL, Li YH, Xu YN, Wang QL, Namgoong S, Cui XS, and Kim NH. 2014. Effects of growth differentiation factor 9 and bone morphogenetic protein 15 on the in vitro maturation of porcine oocytes. Reprod Domest Anim 49:219-227.

Livak KJ, and Schmittgen TD. 2001. Analysis of relative gene expression data using real-time quantitative PCR and the 2(-Delta Delta C(T)) Method. Methods 25:402-408.

Matsuoka A, Kizuka F, Lee L, Tamura I, Taniguchi K, Asada H, Taketani T, Tamura H, and Sugino N. 2010. Progesterone increases manganese superoxide dismutase expression via a cAMP-dependent signaling mediated by noncanonical Wnt5a pathway in human endometrial stromal cells. J Clin Endocrinol Metab 95:E291-299.

Mukherjee A, Malik H, Saha AP, Dubey A, Singhal DK, Boateng S, Saugandhika S, Kumar S, De S, and Guha SK. 2014. Resveratrol treatment during goat oocytes maturation enhances developmental competence of parthenogenetic and hand-made cloned blastocysts by modulating intracellular glutathione level and embryonic gene expression. J Assist Reprod Genet 31:229-239.

Nagyova E, Scsukova S, Kalous J, and Mlynarcikova A. 2014. Effects of RU486 and indomethacin on meiotic maturation, formation of extracellular matrix, and progesterone production by porcine oocyte-cumulus complexes. Domest Anim Endocrinol 48:7-14. 
364

365

366

367

368

369

370

371

372

373

374

375

376

377

378

379

380

381

382

383

384

385

386

387

388

389

390

391

392

393

394

395

396

397

398

399

400

401

402

403

404

Nguyen H, and Syed V. 2011. Progesterone inhibits growth and induces apoptosis in cancer cells through modulation of reactive oxygen species. Gynecol Endocrinol 27:830-836.

Quirk SM, Cowan RG, and Harman RM. 2004. Progesterone receptor and the cell cycle modulate apoptosis in granulosa cells. Endocrinology 145:5033-5043.

Ryan DP, Spoon RA, and Williams GL. 1992. Ovarian follicular characteristics, embryo recovery, and embryo viability in heifers fed high-fat diets and treated with follicle-stimulating hormone. J Anim Sci 70:35053513.

Ryan G, Waddington D, and Campbell K. 1999. Addition of progesterone during bovine oocyte maturation in the presence of gonadotrophins improves developmental competence. Theriogenology 51:392.

Salehnia M, and Zavareh S. 2013. The effects of progesterone on oocyte maturation and embryo development. Int $J$ Fertil Steril 7:74-81.

Salles FJ, and Strickland S. 1999. Analysis of poly(A) tail lengths by PCR: the PAT assay. Methods Mol Biol 118:441-448.

Shimada M, and Terada T. 2002. FSH and LH induce progesterone production and progesterone receptor synthesis in cumulus cells: a requirement for meiotic resumption in porcine oocytes. Mol Hum Reprod 8:612-618.

Shimada M, Yamashita Y, Ito J, Okazaki T, Kawahata K, and Nishibori M. 2004. Expression of two progesterone receptor isoforms in cumulus cells and their roles during meiotic resumption of porcine oocytes. $J \mathrm{Mol}$ Endocrinol 33:209-225.

Sirotkin AV, and Nitray J. 1992. The influence of oxytocin, vasopressin and their analogues on progesterone and testosterone production by porcine granulosa cells in vitro. Ann Endocrinol (Paris) 53:32-36.

Su YQ, Xia GL, Byskov AG, Fu GD, and Yang CR. 1999. Protein kinase C and intracellular calcium are involved in follicle-stimulating hormone-mediated meiotic resumption of cumulus cell-enclosed porcine oocytes in hypoxanthine-supplemented medium. Mol Reprod Dev 53:51-58.

Vannucchi CI, de Oliveira CM, Marques MG, Assumpcao ME, and Visintin JA. 2006. In vitro canine oocyte nuclear maturation in homologous oviductal cell co-culture with hormone-supplemented media. Theriogenology 66:1677-1681.

Wagner GP, Tong Y, Emera D, and Romero R. 2012. An evolutionary test of the isoform switching hypothesis of functional progesterone withdrawal for parturition: humans have a weaker repressive effect of PR-A than mice. J Perinat Med 40:345-351.

Wang HL, Chang ZL, Li KL, Lian HY, Han D, Cui W, Jiao GZ, Wu YG, Luo MJ, and Tan JH. 2011. Caffeine can be used for oocyte enucleation. Cell Reprogram 13:225-232.

Wang WH, Abeydeera LR, Cantley TC, and Day BN. 1997. Effects of oocyte maturation media on development of pig embryos produced by in vitro fertilization. $J$ Reprod Fertil 111:101-108.

Yamashita Y, Kawashima I, Gunji Y, Hishinuma M, and Shimada M. 2010. Progesterone is essential for maintenance of Tace/Adam 17 mRNA expression, but not EGF-like factor, in cumulus cells, which enhances the EGF receptor signaling pathway during in vitro maturation of porcine COCs. $J$ Reprod Dev 56:315-323.

Yang MY, and Rajamahendran R. 2002. Expression of Bcl-2 and Bax proteins in relation to quality of bovine oocytes and embryos produced in vitro. Anim Reprod Sci 70:159-169.

Yoon JD, Jeon Y, Cai L, Hwang SU, Kim E, Lee E, Kim DY, and Hyun SH. 2015. Effects of coculture with cumulus-derived somatic cells on in vitro maturation of porcine oocytes. Theriogenology 83:294-305. 
405

406

407

408

409

410

411

412

413

414

415

416

417

418

419
Zavareh S, Saberivand A, and Salehnia M. 2009. The effect of progesterone on the in vitro maturation and developmental competence of mouse germinal vesicle oocytes. Int J Fertil Steril 3:21-28.

Zhang DX, Cui XS, and Kim NH. 2010. Molecular characterization and polyadenylation-regulated expression of cyclin B1 and Cdc2 in porcine oocytes and early parthenotes. Mol Reprod Dev 77:38-50.

Zhao MH, Jin YX, Lee SK, Kim NH, and Cui XS. 2014. Artificial control maturation of porcine oocyte by dibutyryl cyclicAMP. Animal Cells Syst (Seoul) 18:52-58.

Zheng P. 2007. Effects of in vitro maturation of monkey oocytes on their developmental capacity. Anim Reprod Sci 98:56-71.

Zheng P, Si W, Bavister BD, Yang J, Ding C, and Ji W. 2003. 17Beta-estradiol and progesterone improve in-vitro cytoplasmic maturation of oocytes from unstimulated prepubertal and adult rhesus monkeys. Hum Reprod 18:2137-2144.

Zhou P, Lian HY, Cui W, Wei DL, Li Q, Liu YX, Liu XY, and Tan JH. 2012. Maternal-restraint stress increases oocyte aneuploidy by impairing metaphase I spindle assembly and reducing spindle assembly checkpoint proteins in mice. Biol Reprod 86:83. 
Table $\mathbf{1}$ (on next page)

Primers used for PAT and real-time PCR 
2

\begin{tabular}{cclc}
\hline Gene & GenBank number & \multicolumn{1}{c}{ Primer sequences (5'-3') } & Product size(bp) \\
\hline PAT-Cdc2 & NM_01159304 & CTGTTAACTCTGCTTTTGTCTTGTGT & -- \\
Oligo(dT)-Anchor & -- & GCGAGCTCCGCGGCCGCGT ${ }_{12}$ & -- \\
GAPDH & NM_001206359 & F: GTCGGTTGTGGATCTGACCT & 207 \\
& & R: TTGACGAAGTGGTCGTTGAG & \\
Cdc2 & NM_001159304.2 & F: TAATAAGCTGGGATCTACCACATC & 185 \\
& & R: CGAATGGCAGTACTAGGAACAC & \\
cyclin B1 & NM_001170768.1 & F: AGCTAGTGGTGGCTTCAAGG & 101 \\
& & R: GCGCCATGACTTCCTCTGTA & \\
Bax & XM_005664710.2 & F: GGTCGCGCTTTTCTACTTTG & 111 \\
& & R: CGATCTCGAAGGAAGTCCAG & 193 \\
Bc12 & NM_214285 & F: AGGGCATTCAGTGACCTGAC & 110 \\
& & R: CGATCCGACTCACCAATACC & \\
Casp3 & NM_214131.1 & F: ACTGTGGGATTGAGACGG & \\
& & R: GGAATAGTAACCAGGTGCTG & \\
\hline
\end{tabular}

3

4 
Figure 1 (on next page)

Effect of adding P4 or RU486 on IVM of porcine oocytes

Effect of adding P4 or RU486 on IVM of porcine oocytes. The number of oocytes observed in each experimental group is displayed in the bar. Bars with different superscripted letters (a or $b$ ) in each column indicate statistically significant differences $(p<0.05)$. Values shown are the mean ( \pm standard deviation) of three independent experiments. 


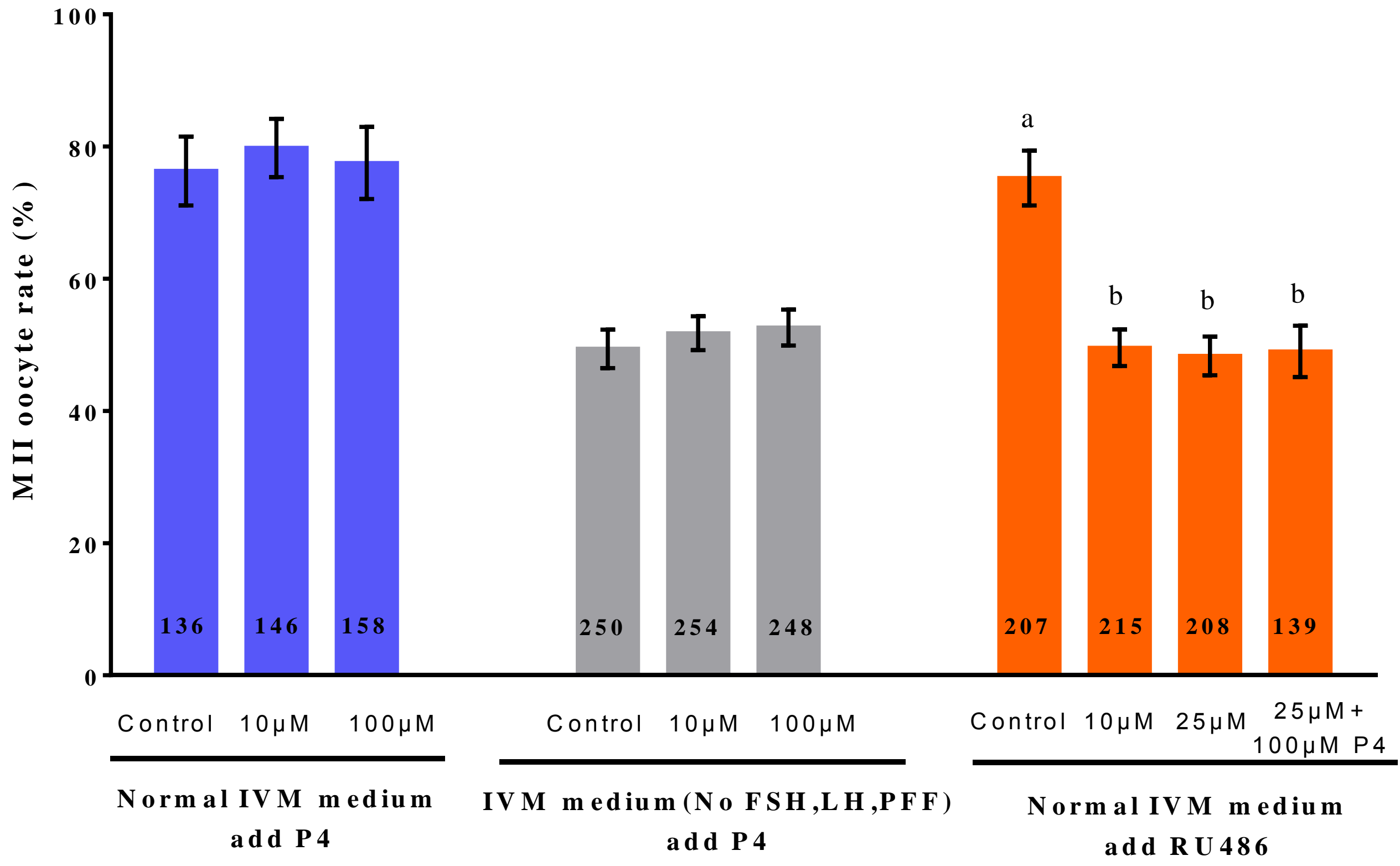


Figure 2 (on next page)

ROS and GSH images of MIl oocytes.

Representative images showing ROS and GSH expression in MIl oocytes. Oocytes were dyed with $\mathrm{H}_{2}$ DCFDA (A-C) and Cell Tracker Blue (D-F) to detect ROS levels and GSH levels. MII oocytes from the control IVM medium and $100 \mu \mathrm{M}$ P4- or $25 \mu \mathrm{M}$ RU486-supplemented IVM system. Effects of P4 or RU486 supplementation during IVM on intracellular ROS and GSH levels in mature oocytes $(G, H)$. Bars with different superscripted letters $(a, b$, and $c)$ in each column indicate statistically significant differences (GSH or ROS; $p<0.05$ ). The experiment was replicated three times. 

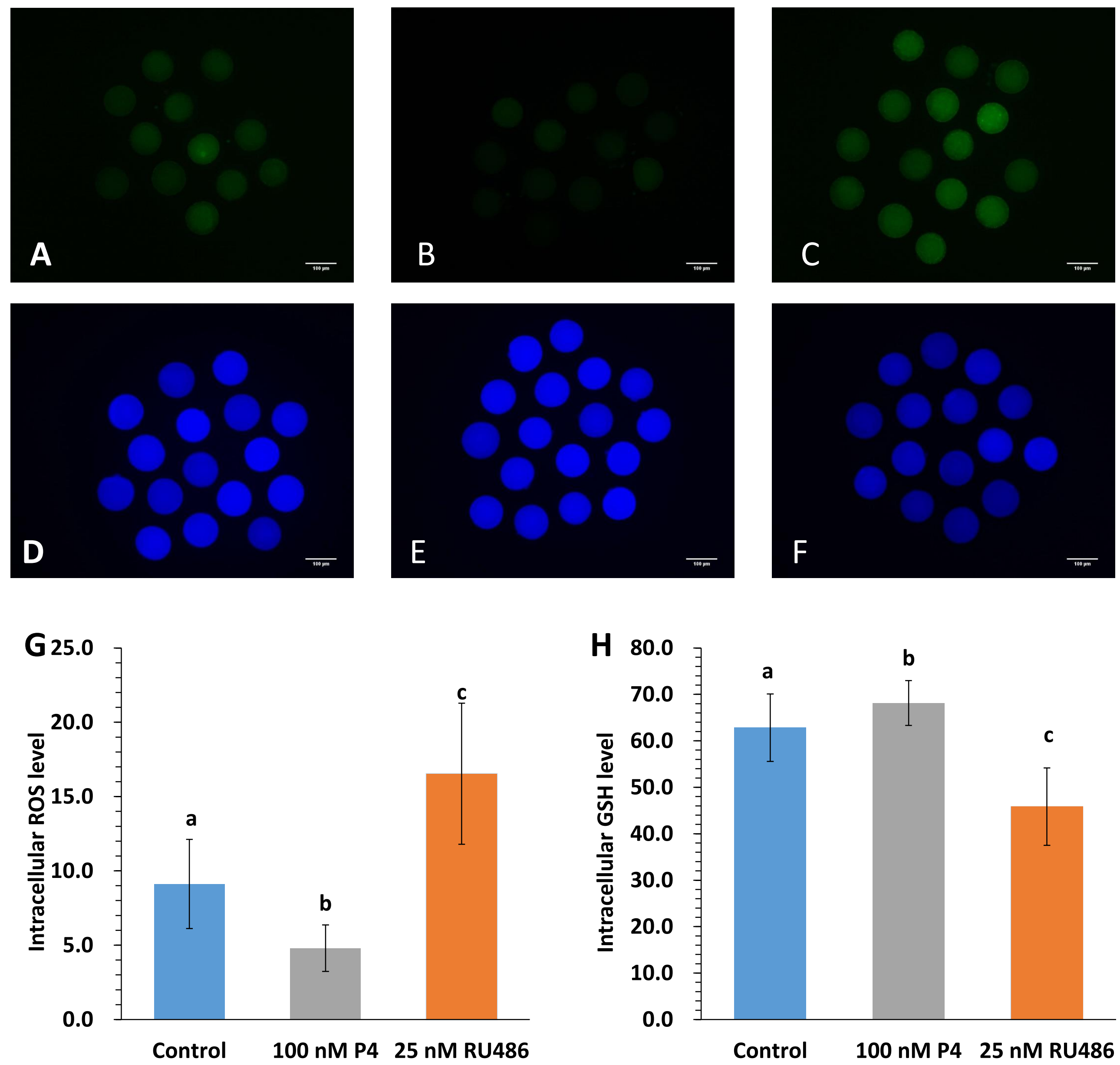


\section{Figure $\mathbf{3}$ (on next page)}

The effects of P4 or RU486 on maternal gene expression, polyadenylation levels and p34cdc2 kinase activity

Maternal mRNA expression at the MII stage (A). Analysis of poly(A) tail length of $C d c 2$ transcripts at the MII stage (B). Treatment with $100 \mu \mathrm{M}$ P4 or $25 \mu \mathrm{M}$ RU486 are indicated at the top of the figure. Oocytes at the MII stage ( $44 \mathrm{~h}$ ). Differences in poly $(A)$ tail lengths of maternal mRNA represented by PCR smears are indicated by dotted lines. MPF activity in MII oocytes (C). MPF was isolated from oocytes treated with $100 \mu \mathrm{M}$ P4 or $25 \mu \mathrm{M}$ RU486. Data are expressed as the percentage \pm SEM of three independent replicates of three experiments. Different superscripted letters show significance $(p<0.05)$. 
A

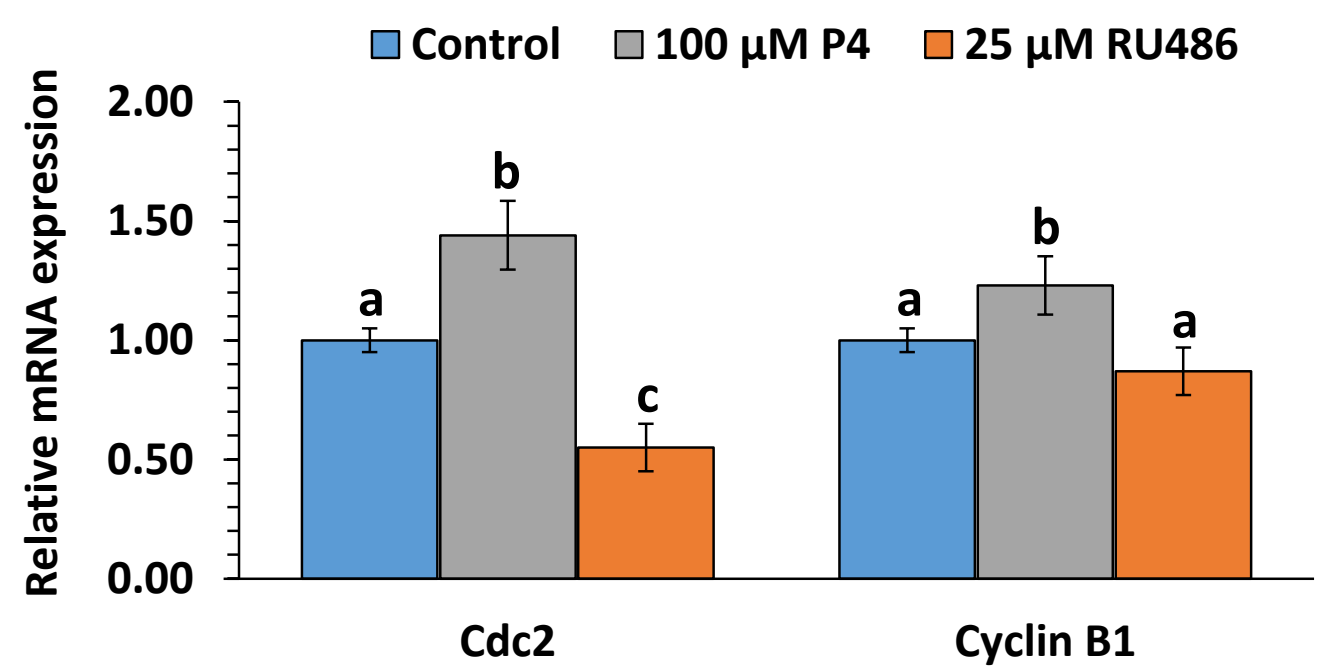

C

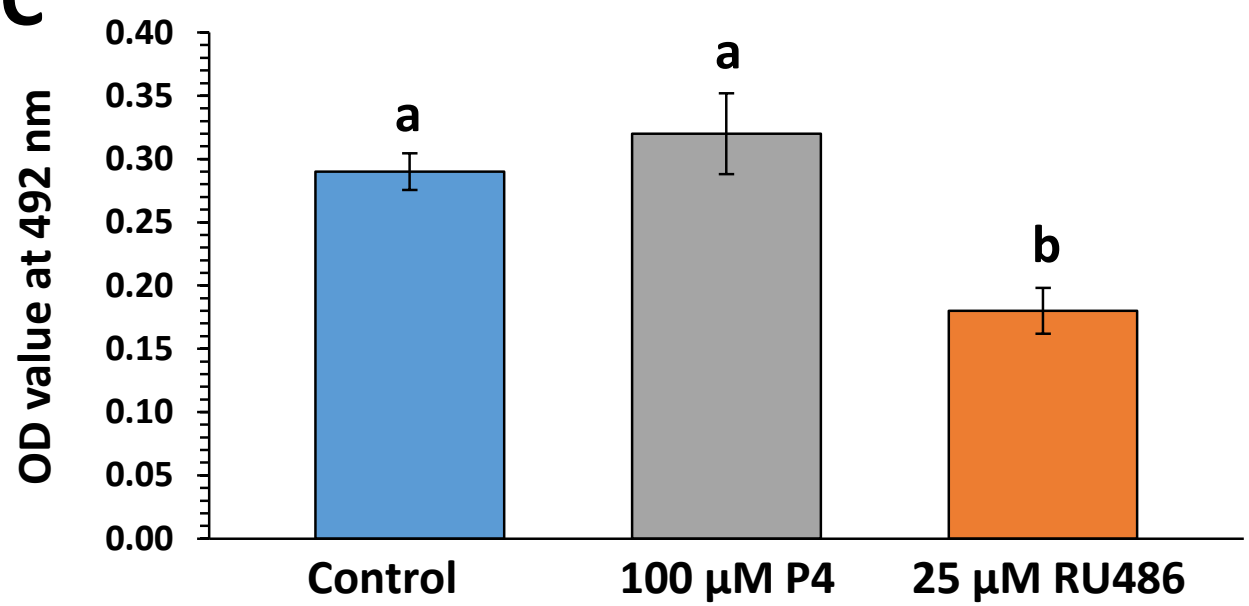

B $M$ Con P4 Ru486

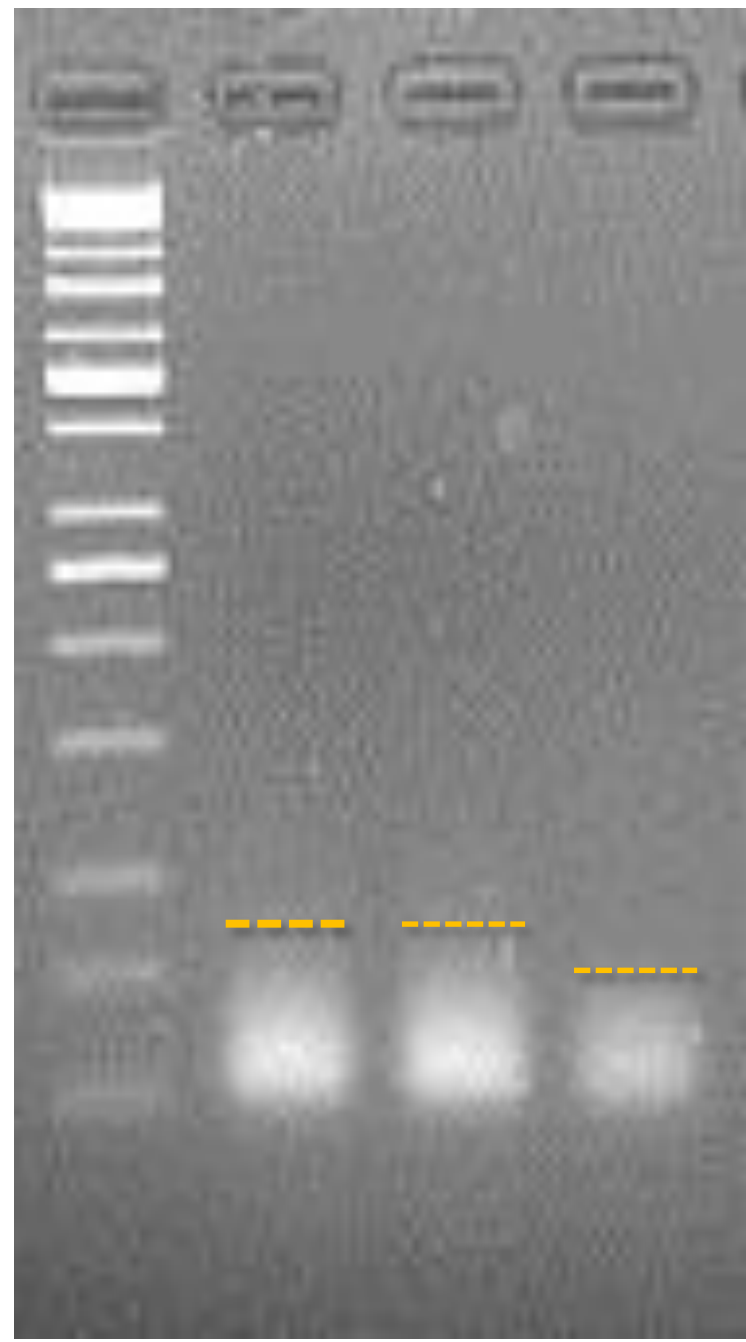


Figure 4(on next page)

Effect of treatment with P4 or RU486 during IVM of porcine oocyte development to the blastocyst stage.

Effect of treatment with P4 or RU486 during IVM on porcine oocyte development to the blastocyst stage. The number of oocytes observed in each experimental group is displayed in the bar. Different letters indicate $p<0.05$. 


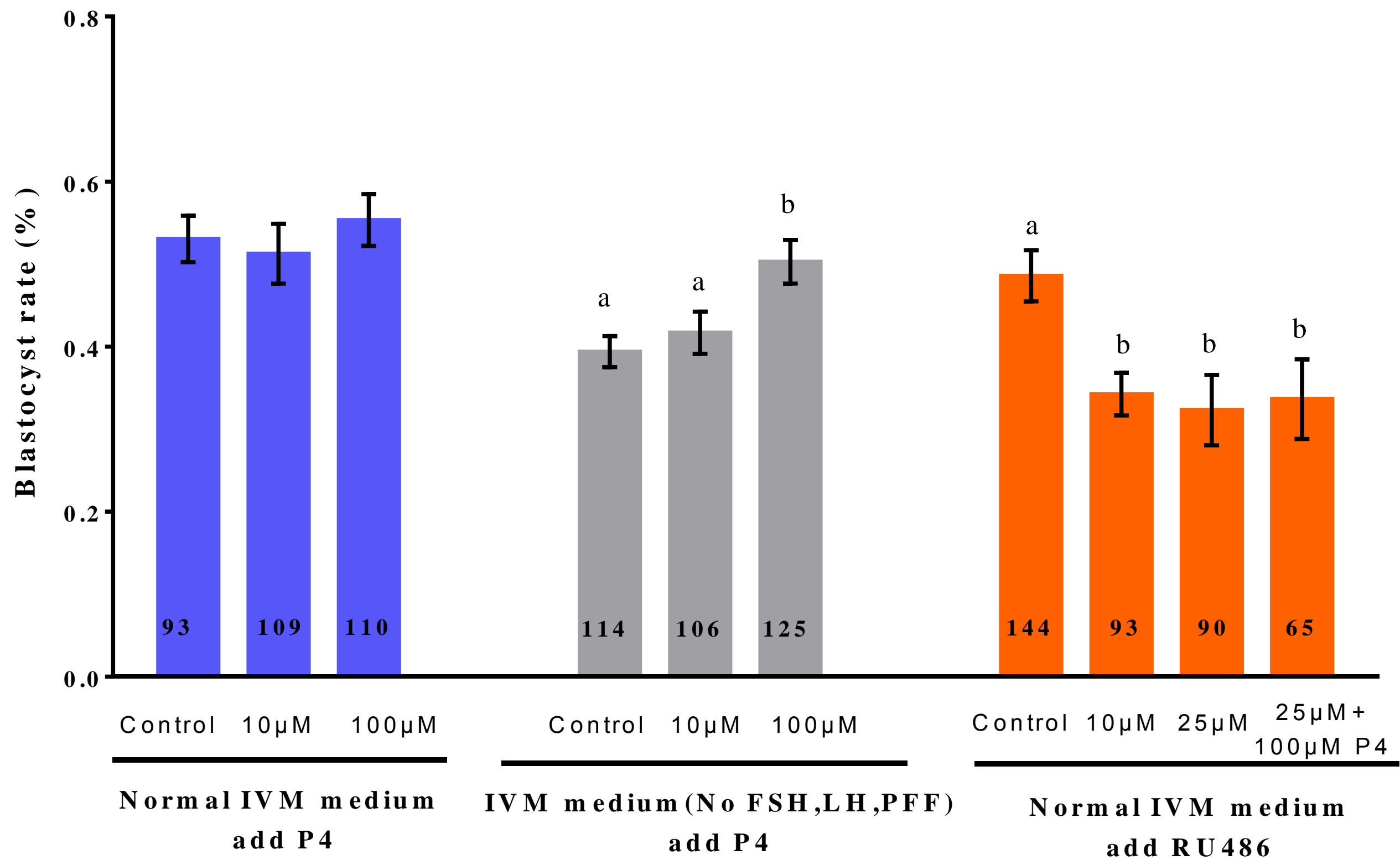


Figure 5 (on next page)

TUNEL assay at blastocyst stage

Representative confocal images of apoptotic cells and nuclear DNA $(\times 400)(A)$. Total numbers of cells per blastocyst in different groups (B). Apoptotic rate per blastocyst in different groups $(C)$. The number of oocytes observed in each experimental group is displayed in the bar. Different letters indicate $p<0.05$. 
Control

$100 \mu \mathrm{M}$

P4

$25 \mu \mathrm{M}$ RU486
Apoptosis

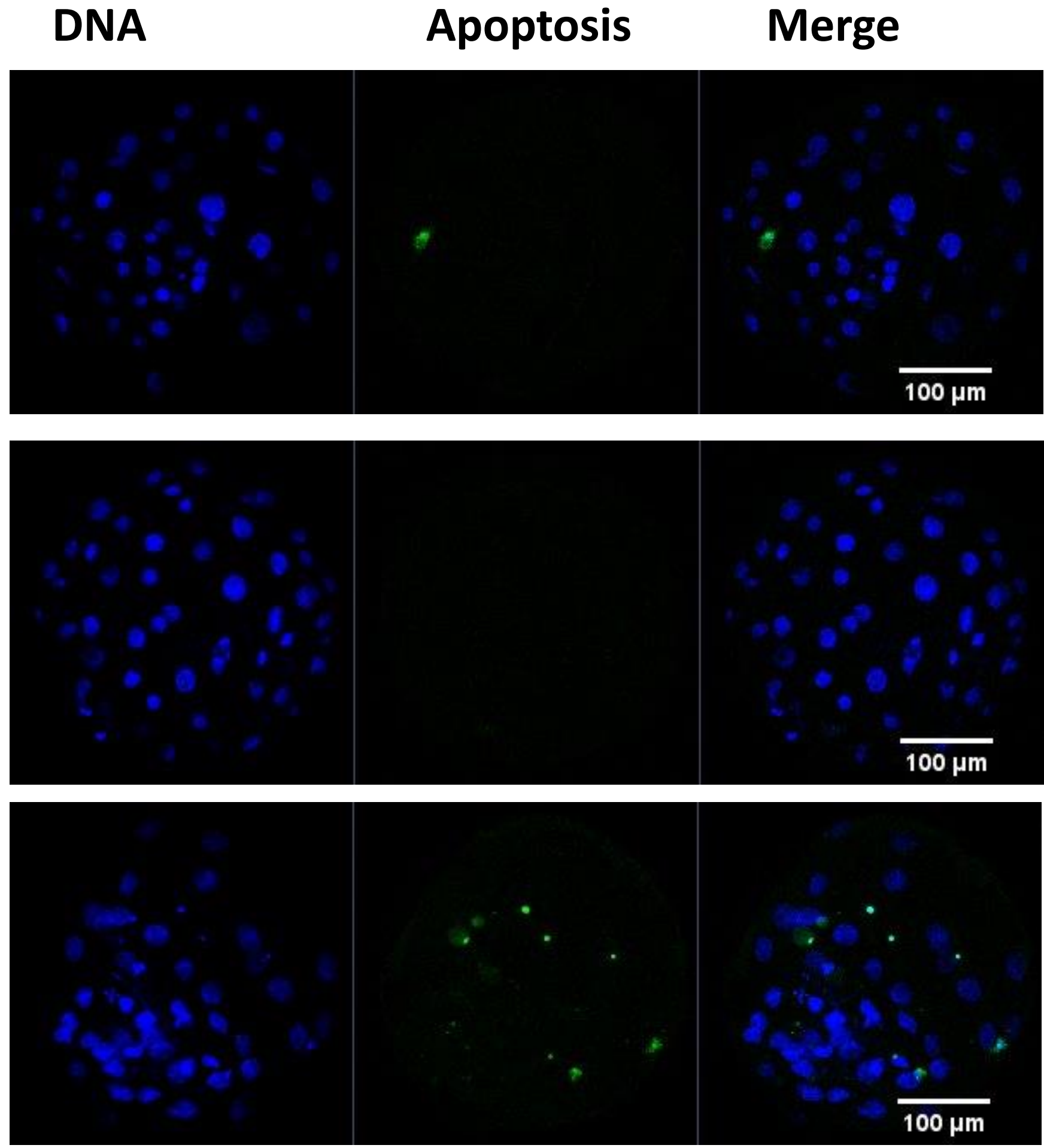

B

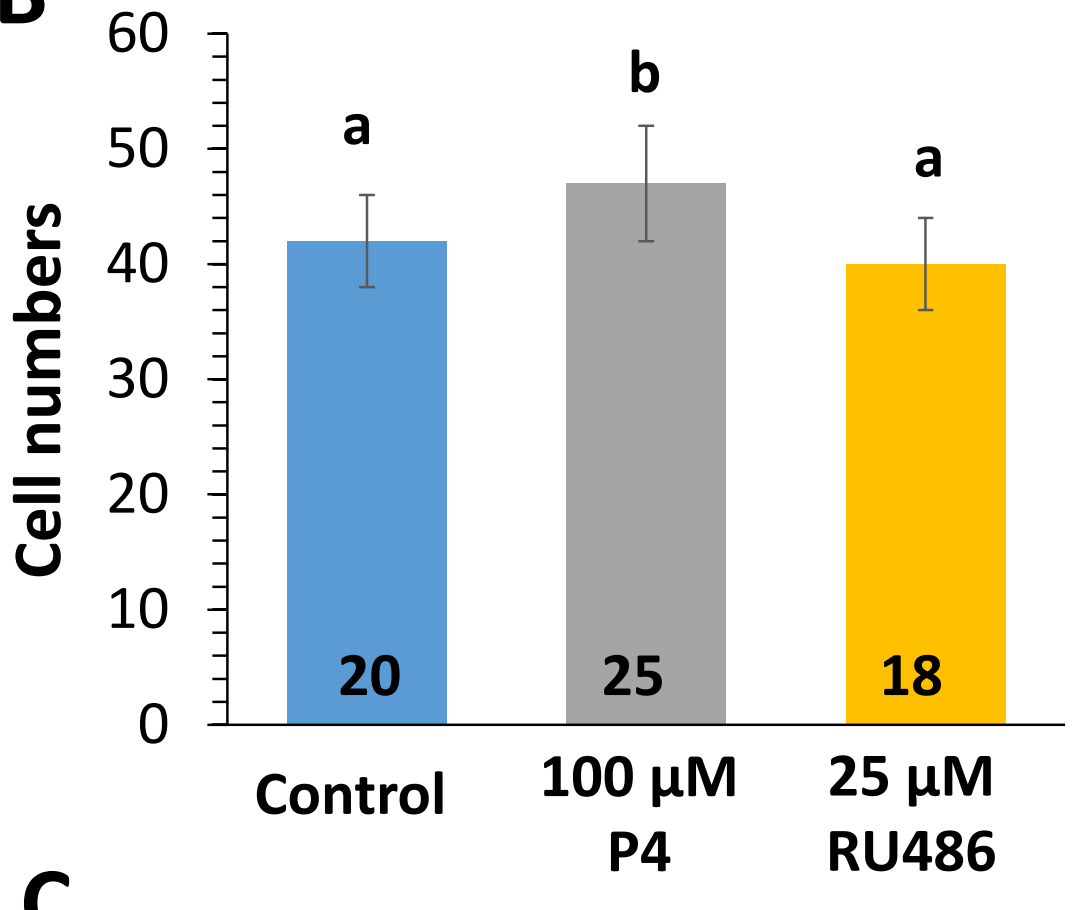

C

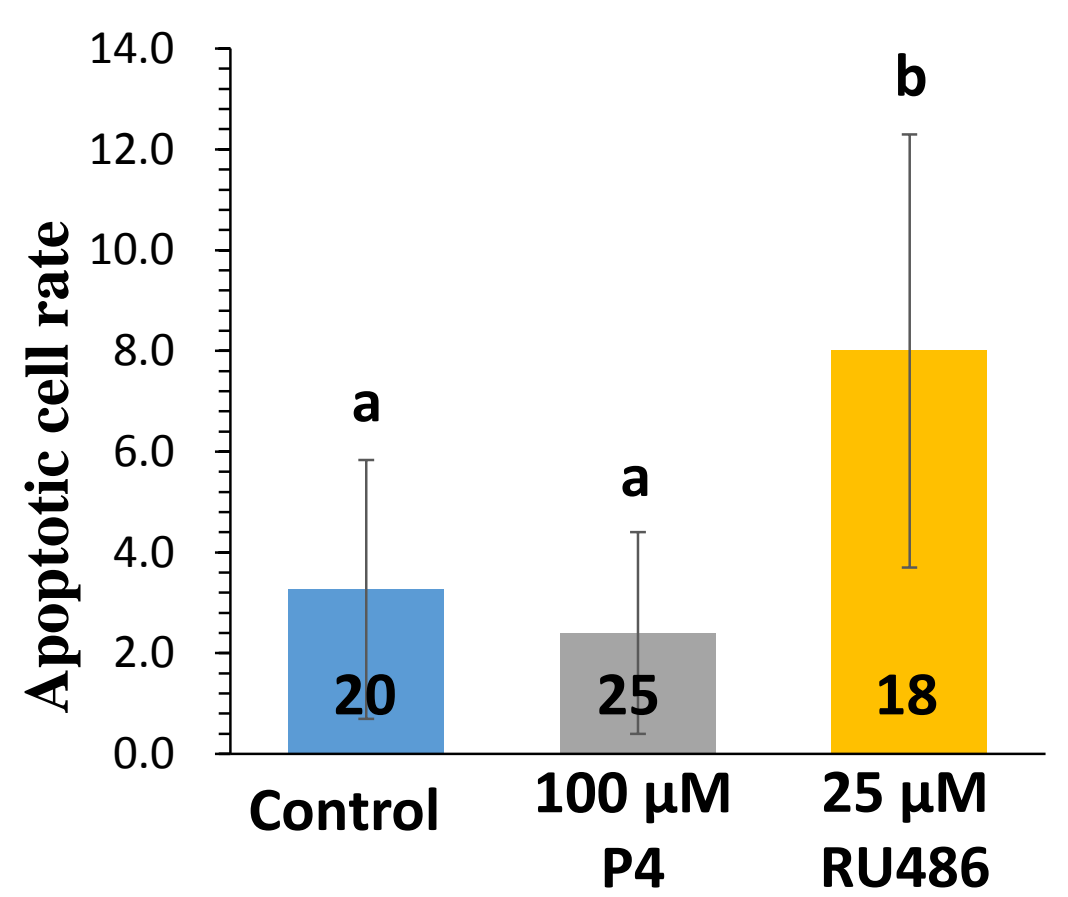




\section{Figure 6 (on next page)}

Effects of P4 or RU486 in IVM on blastocysts apoptosis-related gene expression

BCl-2, Bax, and Casp3 mRNA expression levels in 7-day porcine blastocysts with P4 or RU486 treatment during IVM. In the bars, different letters indicate $p<0.05$. 


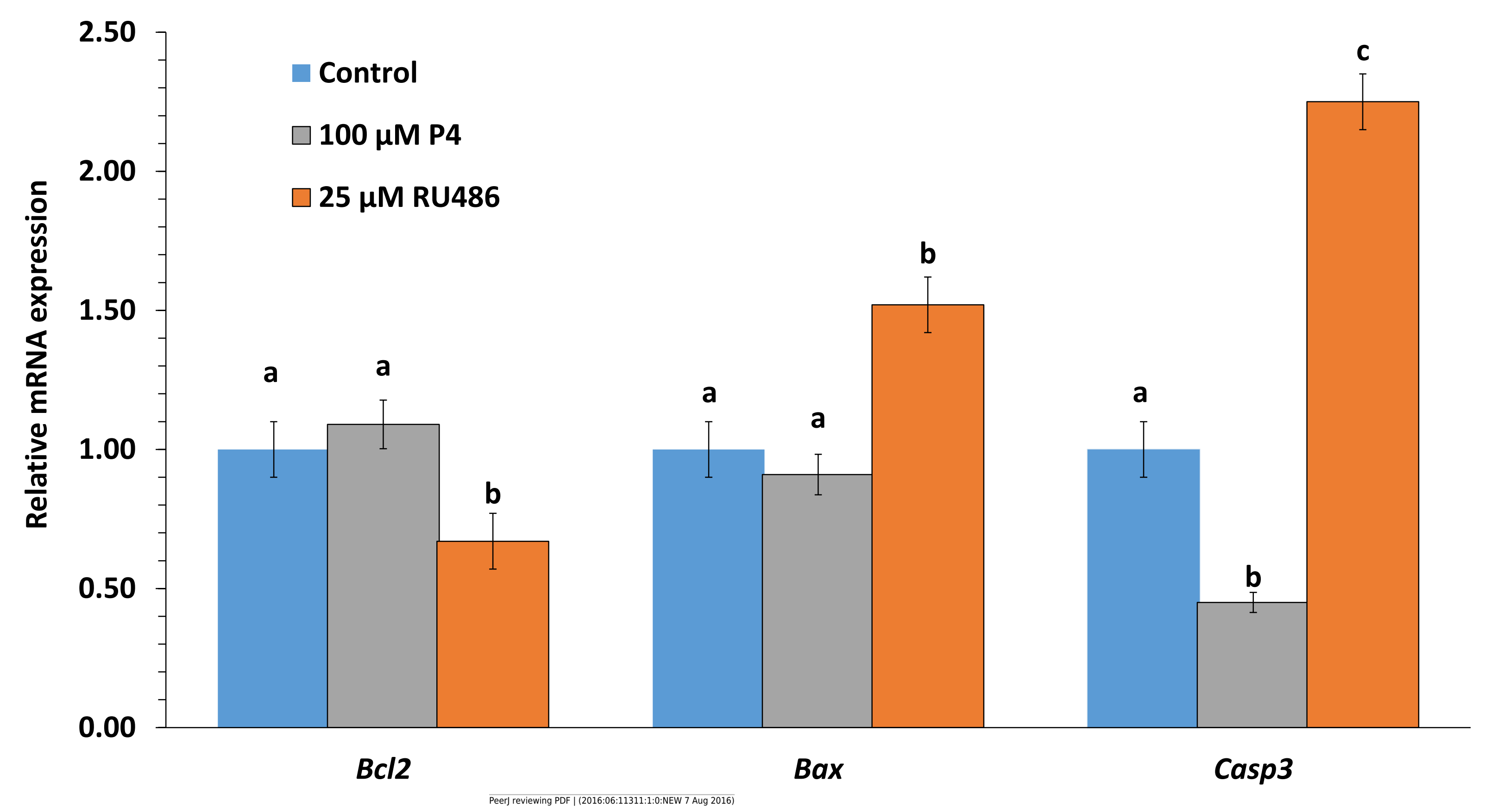

